\title{
Logro de metas de prevención secundaria, prescripción farmacológica y eventos cardiovasculares mayores en pacientes con enfermedad coronaria
}

Facultad de Medicina Universidad de La Frontera, Departamento de Medicina Interna, Unidad de Cardiología, Hospital Dr. Hernán Henríquez Aravena de Temuco.

'Universidad de La Frontera, Temuco, Chile. ${ }^{2}$ Universidad de La

Frontera - Hospital Dr. Hernán Henríquez Aravena, Temuco, Chile.

${ }^{3}$ Hospital Dr. Víctor Ríos Ruiz, Los Ángeles, Chile.

${ }^{\text {aResidente de Medicina }}$ Interna.

Protocolo de investigación financiado por el Departamento de Medicina Interna, Facultad de Medicina, Universidad de La Frontera, Temuco, Chile.

Recibido el 15 de julio de 2012, aceptado el 19 de abril de 2013.

Correspondencia a: Dr. Víctor M. Neira Vidal Universidad de La Frontera, Claro Solar 115, Temuco, Chile.

Teléfono: 045-325755 E-mail: victorneirav@gmail.
VÍCTOR NEIRA V. ${ }^{1}$, MARCELO POTTHOFF N. ${ }^{1, a}$, LUIS QUIÑIÑIR S. ${ }^{1, a}$, GABRIEL LÓPEZ R. ${ }^{1, a}$, BENJAMÍN STOCKINS F. ${ }^{2}$, CARLOS CASTILLO B. ${ }^{3}$, FERNANDO LANAS Z. ${ }^{2}$

\section{Achievement of cardiovascular prevention goals, pharmacologic prescription and major cardiovascular events among patients with coronary disease}

Background: Implementation of health programs to reduce cardiovascular risk, are needed for secondary prevention of cardiovascular diseases. Aim: To evaluate achievements of secondary prevention goals, pharmacologic prescription and major cardiovascular events in patients with coronary artery disease. Material and Methods: Patients who had a first event of acute coronary syndrome, angioplasty or revascularization surgery, between January 2008 and June 2010, were contacted for a clinical and laboratory evaluation between June and October 2011. Results: Seven hundred and two medical records were reviewed and 245 eligible patients were identified. Of these, we assessed 202 patients aged $64 \pm 10$ years (70\% males). Sixty seven percent had two or more cardiovascular risk factors. A goal of HDL cholesterol level over $40 \mathrm{mg} / \mathrm{dL}$ was achieved in $91 \%$ of patients, smoking cessation in $84 \%$ and a total cholesterol $<200 \mathrm{mg} / \mathrm{dL}$ in $66 \%$. A blood pressure below 130/85 $\mathrm{mm} \mathrm{Hg}$ was achieved in 30\% of patients, a normal BMI in 19\% and LDL cholesterol levels below $70 \mathrm{mg} / \mathrm{dL}$ in $18 \%$. At the moment of assessment, $87 \%$ were using aspirin, $78 \%$ statins, $74 \%$ angiotensin converting enzyme inhibitors or angiotensin II receptor antagonists and $66 \%$ were using $\beta$ - blockers. Twenty two percent of patients had a major cardiovascular event during the follow up, which lasted $28 \pm 8$ months. Conclusions: $A$ low percentage of cardiovascular goals achievement was observed in this sample of patients, with a high prevalence of overweight and obesity. A high percentage quit smoking after their first cardiovascular event. Despite the high percentage of drug prescription, hypertension and dyslipidemia were not fully controlled.

(Rev Med Chile 2013; 141: 870-878).

Key words: Coronary artery disease; Secondary prevention.
L a enfermedad coronaria (EC) es la principal causa de mortalidad, discapacidad e ingresos hospitalarios, con altos costos de atención en salud a nivel mundial ${ }^{1,2}$. La prevalencia de EC en el mundo se estima en 100 millones de personas ${ }^{3}$. En Chile, la mortalidad por enfermedad cardiovascu- lar (CV) en el año 2009 representó $27 \%$, siendo un tercio a causa de cardiopatía isquémica ${ }^{4}$.

La prevención secundaria de la EC corresponde a la implementación de programas multifactoriales y multidisciplinarios que abordan la reducción del riesgo $\mathrm{CV}$ y promocionan estilos de vida 
saludables, incluyendo el adecuado manejo de la hipertensión arterial (HTA) e hipercolesterolemia, reducción del consumo de tabaco, los cuales contribuyen a las 3/4 partes de los eventos ${ }^{3}$. Se estima que más de $50 \%$ de los eventos CV podrían evitarse a través del control de estos factores de riesgo $(\mathrm{FR})^{5-7}$.

La prevalencia de factores de riesgo cardiovascular (FRCV) modificables en Latinoamérica es heterogénea, pero las estimaciones por edad y sexo son comparables con otras partes del mundo: tabaquismo $30 \%$ (22-45\%), obesidad 23\% (1827\%), síndrome metabólico 20\% (14-27\%), HTA 18\% (9-29\%), hipercolesterolemia 14\% (6-20\%) y diabetes $7 \%(4-9 \%)^{8}$. Los estudios EUROASPIRE I, II y III demostraron alta prevalencia de FR modificables, que tienen directa implicancia en la salud $\mathrm{CV}^{9-11}$. El beneficio de control de los FRCV se relaciona con el grado de control de estos, en el estudio $\mathrm{STENO}^{12}$ se distribuyeron en forma aleatoria pacientes diabéticos tipo 2 con microalbuminuria a terapia intensiva versus terapia convencional. Hubo una reducción del riesgo de eventos CV cercana al 50\% en el grupo de terapia intensiva, durante el período de seguimiento. Por lo tanto, la etiología multifactorial de las enfermedades CV hacen necesarias múltiples intervenciones para maximizar el efecto de la atención preventiva ${ }^{11,13,14}$.

El objetivo de nuestro estudio fue evaluar el logro de metas CV en pacientes con EC, de acuerdo a las metas establecidas por el Programa de Salud Cardiovascular implementado por el Ministerio de Salud - Chile ${ }^{14}$, en una población de pacientes que egresaron vivos del Hospital Dr. Hernán Henríquez Aravena de Temuco (HHA), entre enero de 2008 y junio de 2010 .

\section{Material y Método}

Estudio de corte transversal. Selección de pacientes que cursaron con un primer evento secundario a EC, entre enero de 2008 y junio de 2010, a partir del banco de datos del Hospital HHA de Temuco. Se identificó a aquellos procedentes de Temuco y vivos al momento del estudio, que cumplían con criterios de inclusión. Se contactaron telefónicamente y se citaron entre junio y octubre de 2011, para la aplicación de una encuesta sociodemográfica y evaluación del logro de metas en cuanto a FRCV, prescripción farmacológica y eventos CV mayores durante el período de seguimiento. Se realizó además medición de peso, talla, perímetro de cintura (PC), presión arterial (PA) y toma de muestra de sangre en ayunas para determinar perfil lipídico, creatininemia, glicemia y en pacientes diabéticos hemoglobina glicosilada.

Para evaluar el logro de metas se utilizaron los objetivos establecidos por el Programa de Salud Cardiovascular implementado por el Ministerio de Salud-Chile: abandono del tabaquismo, PA $<130 / 85 \mathrm{~mm} \mathrm{Hg}$, colesterol total (CT) $<200 \mathrm{mg} /$ $\mathrm{dL}$, triglicéridos $<150 \mathrm{mg} / \mathrm{dL}$, colesterol HDL $>40 \mathrm{mg} / \mathrm{dL}$, colesterol LDL $<100 \mathrm{mg} / \mathrm{dL}$ (deseable $<70 \mathrm{mg} / \mathrm{dL}$ ), hemoglobina glicosilada $<7 \%$, índice de masa corporal (IMC) normal (18,5-24,9 $\left.\mathrm{kg} / \mathrm{m}^{2}\right), \mathrm{PC}<102 \mathrm{~cm}$ en hombres y $<88 \mathrm{~cm}$ en mujeres y prescripción de fármacos de acción $\mathrm{CV}$ en dosis adecuadas ${ }^{14}$. Los eventos CV mayores fueron: angioplastía coronaria percutánea (ACP) de urgencia o electiva, cirugía de revascularización miocárdica (CRM) electiva o de urgencia, nuevo infarto agudo al miocardio (IAM) con elevación del segmento ST, síndrome coronario agudo (SCA) sin elevación del segmento ST y accidente cerebro vascular (ACV).

Todos los participantes firmaron un consentimiento informado aprobado por el Comité de Ética Científico del Servicio de Salud Araucanía Sur.

\section{Tamaño muestral}

Se calculó asumiendo una prevalencia de un FR de $40 \%$ y una precisión estimada de $7 \%$, con un IC de $95 \%$, con lo cual el tamaño muestral estimado es de 189 sujetos.

\section{Criterios de inclusión}

1. Pacientes atendidos en el Hospital HHA y residentes de la ciudad de Temuco.

\section{Mayores de 18 y menores de 80 años.}

3. Los criterios de inclusión para pacientes con EC fueron similares a los criterios del EUROASPIRE I, con número similar de pacientes por grupo, con fines de comparación de resultados.

a. Cirugía de revascularización miocárdica: pacientes consecutivos con primera CRM electiva o de emergencia, sin antecedentes de recambio valvular.

b. Angioplastía coronaria percutánea: pacientes consecutivos con primera ACP electiva o de emergencia, sin antecedentes de CRM. 
c. Infarto agudo al miocardio con supradesnivel del segmento ST: pacientes consecutivos que ingresaron con diagnóstico de IAM (primer evento o recurrente), sin antecedentes de ACP o CRM.

d. Síndrome coronario agudo sin elevación del segmento ST: pacientes consecutivos que ingresaron con diagnóstico de isquemia aguda al miocardio (primer evento o recurrente), sin antecedentes de IAM, CRM o ACP.

\section{Análisis de datos}

Las variables fueron ingresadas a una base de datos diseñada en Epi Info ${ }^{\circledR}$ 3.5.3. La validación de datos y análisis estadísticos fueron realizados en el programa Stata ${ }^{\circledR} 10.1$.

Se realizó una descripción de los pacientes en cuanto a características sociodemográficas, prevalencia de FR, tratamiento prescrito al alta posterior al primer evento coronario que determinó la elegibilidad y recibido al momento del control e incidencia de eventos CV mayores durante el período de seguimiento. Se estimó el valor promedio y desviación estándar para variables continuas. Se utilizó T test para comparar variables continuas y prueba de $\chi^{2}$ para variables categóricas. Con los puntos de corte establecidos en las recomendaciones, se determinó la proporción de sujetos que alcanzaron dichas metas. Para evaluar un posible sesgo de selección, se compararon aquellos pacientes que no se lograron controlar con los sujetos controlados y no hubo diferencias estadísticamente significativas.

Para ponderar el cumplimiento de cada una de las metas estudiadas, se modeló el número de metas cumplidas en relación al logro de cada una de ellas a través de un análisis de regresión binomial con método de estimación basado en los scores de Fisher.

\section{Resultados}

Se revisaron 702 historias clínicas durante el período señalado, se aplicaron criterios de inclusión; $75(10,6 \%)$ pacientes del total del grupo fallecieron; 245/702 pacientes fueron elegibles. De los pacientes elegibles, $38(15,5 \%)$ pacientes no desearon participar y $5(2,0 \%)$ no fueron posibles de contactar.

Los FRCV previos al primer evento coronario fueron: HTA, 81,6\%; tabaquismo, 50,4\%; diabetes mellitus tipo $2,34,6 \%$; antecedente de dislipidemia, 28,7\%; enfermedad renal crónica, 1,9\%. La prescripción de fármacos previo al primer evento coronario fue: inhibidores de enzima convertidora (IECA) o antagonistas de los receptores de angiotensina II (ARAII), 50,0\%; ácido acetilsalicílico, $41,5 \%$; estatinas, $25,7 \%$ y beta bloqueadores adrenérgicos, $17,8 \%$. El 35,7\% de los pacientes con diabetes mellitus tipo 2 estaba en tratamiento regular.

Se controlaron 202 pacientes. Sus características sociodemográficas al momento del control clínico se describen en la Tabla 1, en cuanto a edad, sexo, estado civil, nivel de escolaridad, tramo de ingreso mensual familiar y promedio de personas en el hogar.

La Tabla 2 describe los FRCV al control, donde destaca la HTA $(86,5 \%)$ como el FR más frecuente, antecedente de dislipidemia, $36,2 \%$ y diabetes mellitus tipo 2 en 35,6\%. Cincuenta coma cinco por ciento fumaba en promedio $21,7 \pm 20,1$ paquetes/ año previo al primer evento coronario, y posterior a este, $84,3 \%$ de estos fumadores, abandonó el hábito tabáquico. Sólo 7,9\% actualmente fuma, en promedio $19,0 \pm 12,3$ paquetes/año. En relación a la evaluación nutricional, la mayoría de los

\section{Tabla 1. Características sociodemográficas}

\begin{tabular}{|lc|}
\hline $\begin{array}{l}\text { Variables } \\
\text { demográficas }\end{array}$ & $\begin{array}{c}\text { Promedios-frecuencias } \\
\text { (\%) }\end{array}$ \\
\hline Edad (años) & $\begin{array}{c}63,7 \pm 9,6 \\
\text { (rango } 36-79 \text { años) }\end{array}$ \\
\hline Sexo femenino & $29,7 \%$ \\
\hline Edad sexo femenino (años) & $66,0 \pm 8,6$ \\
\hline Edad sexo masculino (años) & $62,8 \pm 9,9$ \\
\hline Estado civil, casado & $68,2 \%$ \\
\hline Nivel escolaridad & Analfabetismo \\
• Enseñanza básica & $9,4 \%$ \\
• Enseñanza media & $37,1 \%$ \\
\hline Trabajo remunerado & $36,1 \%$ \\
\hline $\begin{array}{l}\text { Ingreso mensual familiar } \\
\text { \$100.000 - 300.000 }\end{array}$ & $17,4 \%$ \\
\hline $\begin{array}{l}\text { Promedio personas en el } \\
\text { hogar (n) }\end{array}$ & $45,0 \%$ \\
\hline
\end{tabular}


Tabla 2. Factores de riesgo cardiovascular al momento de la evaluación

\begin{tabular}{|c|c|}
\hline Factores de riesgo cardiovascular & $\begin{array}{c}\text { Frecuencia } \\
\text { (\%) }\end{array}$ \\
\hline Hipertensión arterial crónica & 86,5 \\
\hline Antecedente de dislipidemia* & 36,2 \\
\hline Diabetes Mellitus tipo 2 & 35,6 \\
\hline \multicolumn{2}{|l|}{ Índice de masa corporal } \\
\hline - Normal $\quad(18,5-24,9$ kg/m²) & 17,4 \\
\hline - Sobrepeso $(25,0-29,9$ kg/m²) & 51,4 \\
\hline - Obesidad $\quad\left(\geq 30 \mathrm{~kg} / \mathrm{m}^{2}\right)$ & 31,2 \\
\hline \multicolumn{2}{|l|}{ Tabaquismo } \\
\hline - Nunca & 49,5 \\
\hline - Ex-fumador & 42,6 \\
\hline - Fumador & 7,9 \\
\hline $\begin{array}{l}\text { Clearence creatininemia } \\
<60 \mathrm{ml} / \mathrm{min} / 1,73 \mathrm{~m}^{2 * *}\end{array}$ & 10,8 \\
\hline $\begin{array}{l}\text { Antecedentes familiares de enfermedad } \\
\text { coronaria precoz }{ }^{\dagger}\end{array}$ & 12,3 \\
\hline
\end{tabular}

*Antecedente de dislipidemia obtenido de registro en ficha clínica. ${ }^{* *}$ Clearence de creatininemia estimado según fórmula de Crockcroft y Gault. 'Familiar de primer grado, menor de 55 años si es hombre o menor de 65 años si es mujer. pacientes correspondió a sobrepeso u obesidad. Se interrogó acerca de su situación clínica actual: $71,7 \%$ estaban asintomáticos, $24,3 \%$ con angor de esfuerzo y $3,9 \%$ con angor de reposo.

En relación al tratamiento farmacológico (Tabla 3) y su frecuencia de prescripción, no hay diferencias estadísticamente significativas en la distribución por años, salvo en el uso de diuréticos $(\mathrm{p}=0,005)$.

En cuanto al cumplimiento de las metas CV del grupo total (Tabla 4), lo más frecuente fue el colesterol HDL $>40 \mathrm{mg} / \mathrm{dL}$ en $91,5 \%$, abandono de tabaquismo $84,4 \%$ y CT $<200 \mathrm{mg} / \mathrm{dL}$ en $65,8 \%$. El menor cumplimiento se observó en la obtención de $\mathrm{PA}<130 / 85 \mathrm{~mm} \mathrm{Hg}(30,2 \%)$, colesterol $\mathrm{LDL}<70 \mathrm{mg} / \mathrm{dL}(18,3 \%)$ e IMC normal $(18,8 \%)$. Con respecto a los pacientes que no logran metas de PA, son más añosos en relación a los que sí la logran, $61,9 \pm 1,3$ vs $54,5 \pm 9,3$ años $(\mathrm{p}=0,03)$. El 8,6\% no recibía tratamiento por abandono de controles médicos o por falta de adherencia al tratamiento, $40 \%$ eran diabéticos, $60,1 \%$ recibe 2-3 fármacos antihipertensivos (IECA o ARAII, asociado a diurético o beta bloqueador adrenérgico), en dosis subóptimas. No hubo diferencias estadísticamente significativas en la distribución de los grupos por año.

En cuanto a los factores asociados al logro de metas CV tales como: sexo, edad, estado civil,

Tabla 3. Prescripción de tratamiento farmacológico al momento del control, según año del primer evento coronario que determinó la elegibilidad

\begin{tabular}{|c|c|c|c|c|c|}
\hline Tratamiento farmacológico & $\begin{array}{c}\text { Grupo } 2008 \\
(n=89)\end{array}$ & $\begin{array}{c}\text { Grupo } 2009 \\
(n=82)\end{array}$ & $\begin{array}{c}\text { Grupo } 2010 \\
(n=31)\end{array}$ & $\begin{array}{c}\text { Total } \\
(\mathrm{n}=\mathbf{2 0 2})\end{array}$ & Valor de $p$ \\
\hline Aspirina & $86,5 \%(77)$ & $83,3 \%(70)$ & $90,3 \%(28)$ & $86,6 \%(175)$ & 0,85 \\
\hline Estatinas & $79,8 \%(71)$ & $74,3 \%(61)$ & $80,6 \%(25)$ & $77,7 \%(157)$ & 0,66 \\
\hline Bloqueadores $\beta$ adrenérgicos & $67,4 \%(60)$ & $64,6 \%(53)$ & $70,9 \%(22)$ & $66,3 \%(135)$ & 0,82 \\
\hline $\mathrm{IECA}^{\dagger}$ y ARAII ${ }^{\dagger+}$ & $77,3 \%(69)$ & $69,5 \%(57)$ & $74,1 \%(23)$ & $73,7 \%(149)$ & 0,50 \\
\hline Diuréticos & $37,0 \%(33)$ & $18,2 \%(15)$ & $12,9 \% \quad(4)$ & $25,7 \% \quad(52)$ & 0,005 \\
\hline Clopidogrel & $15,7 \%(14)$ & $24,3 \%(20)$ & $22,5 \% \quad(7)$ & $20,3 \% \quad(41)$ & 0,33 \\
\hline Antagonista canales calcio & $15,7 \%(14)$ & $12,1 \%(10)$ & $12,9 \% \quad(4)$ & $13,8 \% \quad(28)$ & 0,82 \\
\hline Espironolactona & $8,9 \% \quad(8)$ & $12,2 \%(10)$ & $16,3 \% \quad(5)$ & $11,3 \% \quad(23)$ & 0,49 \\
\hline $\begin{array}{l}\text { Diabetes Mellitus tipo } 2 \\
\text { - Dieta } \\
\text { - Hipoglicemiantes orales } \\
\text { - Insulina }\end{array}$ & $\begin{array}{l}64,5 \%(20) \\
20,9 \% \quad(9) \\
28,0 \%(25)\end{array}$ & $\begin{array}{l}63,3 \%(19) \\
33,3 \%(10) \\
31,7 \%(26)\end{array}$ & $\begin{array}{ll}63,6 \% & (7) \\
27,2 \% & (3) \\
25,8 \% & (8)\end{array}$ & $\begin{array}{ll}63,8 \% & (46) \\
30,5 \% & (22) \\
29,2 \% & (54)\end{array}$ & $\begin{array}{l}1,0 \\
0,94 \\
0,83\end{array}$ \\
\hline
\end{tabular}

${ }^{\dagger} \mathrm{IECA}=$ inhibidores de la enzima convertidora de angiotensina; ${ }^{\dagger \dagger}$ ARAll = antagonista de los receptores de angiotensina $\mathrm{I}$. 
Logro de metas de prevención secundaria en pacientes con enfermedad coronaria - V. Neira et al

Tabla 4. Logro de metas cardiovasculares al momento del control, según año del primer evento coronario que determino la elegibilidad

\begin{tabular}{|c|c|c|c|c|c|}
\hline $\begin{array}{l}\text { Metas cardiovasculares - } \\
\text { frecuencia cumplimiento (\%) }\end{array}$ & $\begin{array}{l}\text { Grupo } 2008 \\
\quad(n=89)\end{array}$ & $\begin{array}{l}\text { Grupo } 2009 \\
\quad(n=82)\end{array}$ & $\begin{array}{l}\text { Grupo } 2010 \\
(n=31)\end{array}$ & $\begin{array}{c}\text { Total } \\
(n=202)\end{array}$ & Valor de $p$ \\
\hline Meta PS $<130 \mathrm{~mm} \mathrm{Hg}^{\dagger}$ & $29,2 \%(26)$ & $34,1 \%(28)$ & $32,2 \%(10)$ & $31,6 \% \quad(64)$ & 0,78 \\
\hline Meta PD $<85 \mathrm{~mm} \mathrm{Hg}^{\dagger+}$ & $64,0 \%(57)$ & $63,4 \%(52)$ & $70,9 \%(22)$ & $64,8 \%(131)$ & 0,70 \\
\hline Meta PA $<130 / 85$ mm Hg ${ }^{*}$ & $24,7 \%(22)$ & $35,3 \%(29)$ & $32,2 \%(10)$ & $30,2 \% \quad(61)$ & 0,30 \\
\hline IMC normal $\left(18,5-24,9 \mathrm{~kg} / \mathrm{m}^{2}\right)^{* *}$ & $22,4 \%(20)$ & $17,0 \%(14)$ & $12,9 \% \quad(4)$ & $18,8 \% \quad(38)$ & 0,49 \\
\hline Sexo femenino $\mathrm{PC}<88 \mathrm{~cm}^{\S}$ & $12,0 \%$ & $20,0 \%$ & $19,0 \% \quad(1)$ & $15,0 \%$ & 0,71 \\
\hline Sexo masculino $\mathrm{PC}<102 \mathrm{~cm}^{\S}$ & $60,9 \%(39)$ & $68,4 \%(39)$ & $52,3 \%(11)$ & $62,6 \% \quad(89)$ & 0,43 \\
\hline Hemoglobina glicosilada $<7 \%$ & $48,1 \%(13)$ & $50,0 \%(13)$ & $54,5 \% \quad(6)$ & $50,0 \% \quad(32)$ & 1,0 \\
\hline Abandono tabaquismo & $93,0 \%(40)$ & $78,5 \%(33)$ & $77,7 \%(14)$ & $84,4 \% \quad(87)$ & 0,12 \\
\hline Colesterol total $<200 \mathrm{mg} / \mathrm{dL}$ & $62,9 \%(56)$ & $65,8 \%(54)$ & $74,1 \%(23)$ & $65,8 \%(133)$ & 0,52 \\
\hline Triglicéridos < 150 mg/dL & $53,4 \%(48)$ & $57,3 \%(47)$ & $41,9 \%(13)$ & $53,4 \%(108)$ & 0,34 \\
\hline Colesterol LDL < $100 \mathrm{mg} / \mathrm{dL}$ & $46,0 \%(41)$ & $57,3 \%(47)$ & $51,6 \%(16)$ & $51,4 \%(104)$ & 0,35 \\
\hline Colesterol LDL $<70$ mg/dL & $17,9 \%(16)$ & $17,5 \%(14)$ & $22,5 \% \quad(7)$ & $18,3 \% \quad(37)$ & 0,80 \\
\hline Colesterol HDL > 40 mg/dL & $94,3 \%(84)$ & $89,0 \%(73)$ & $90,3 \%(28)$ & $91,5 \%(185)$ & 0,37 \\
\hline
\end{tabular}

${ }^{\dagger} \mathrm{PS}=$ presión sistólica; ${ }^{\dagger+} \mathrm{PD}=$ presión diastólica; ${ }^{*} \mathrm{PA}=$ presión arterial; ${ }^{*} \mathrm{IMC}=$ índice de masa corporal; ${ }^{\$} \mathrm{PC}=$ perímetro de cintura.

ingreso familiar, nivel educacional, tipo de evento que determinó la elegibilidad (ACP, CRM, IAM con elevación de ST, SCA sin elevación ST) y número de FRCV, se realizó análisis de estas variables las cuales no fueron estadísticamente significativas.

Durante el período de seguimiento, los pacientes tuvieron en promedio $5,1 \pm 3,9$ controles médicos (rango 0-20 controles) y al alta fueron educados por la enfermera del Programa Cardiovascular del hospital. Se realizó un análisis entre el número de controles médicos y el cumplimiento de metas, donde no se encontraron diferencias estadísticamente significativas.

Del total de pacientes, 22,2\% presentaron al menos un evento cardiovascular mayor (Figura 1), durante un seguimiento promedio de 28,0 $\pm 8,3$ meses. Al analizar los FRCV de este grupo (Tabla 5) con respecto a los pacientes que no presentaron eventos CV mayores destaca que este último grupo tiene menor porcentaje de antecedente de dislipidemia ( 52,2 vs $73,3 \%$ ); y con respecto al cumplimiento de metas $\mathrm{CV}$ entre ambos grupos no hubieron diferencias estadísticamente significativas. Posterior al evento coronario índice, 7,9\% de los sujetos presentó un evento CV mayor durante el primer año, los cuales en su mayoría fueron: ACP de urgencia $2,4 \%$, CRM 1,4\% y nuevo IAM con elevación de ST 1,0\%. La incidencia de eventos CV mayores según el evento coronario que determinó la elegibilidad fueron: CRM 14,0\%, tiempo promedio 16,2 $\pm 10,6$ meses; SCA sin elevación de ST $17,6 \%$, tiempo promedio $13,5 \pm 9,6$ meses; ACP $28,0 \%$, tiempo promedio $14,6 \pm 9,5$ meses e IAM con elevación de ST 29,0\%, tiempo promedio 13,7 $\pm 8,4$ meses. En la Tabla 7 se detallan los eventos CV mayores durante el período de seguimiento. $\mathrm{Al}$ analizar los eventos $\mathrm{CV}$ mayores en pacientes diabéticos versus no diabéticos no se observaron diferencias en su incidencia.

\section{Discusión}

Los resultados de nuestro estudio muestran un bajo grado de cumplimento de las metas $\mathrm{CV}$ establecidas para controlar los FRCV, de acuerdo a las normas del Ministerio de Salud ${ }^{14}$.

La prevalencia de obesidad y sobrepeso en la población estudiada es alta. Esto se ha observado en otros estudios poblacionales y se correlaciona con la tendencia epidemiológica mundial ${ }^{15-17}$. 


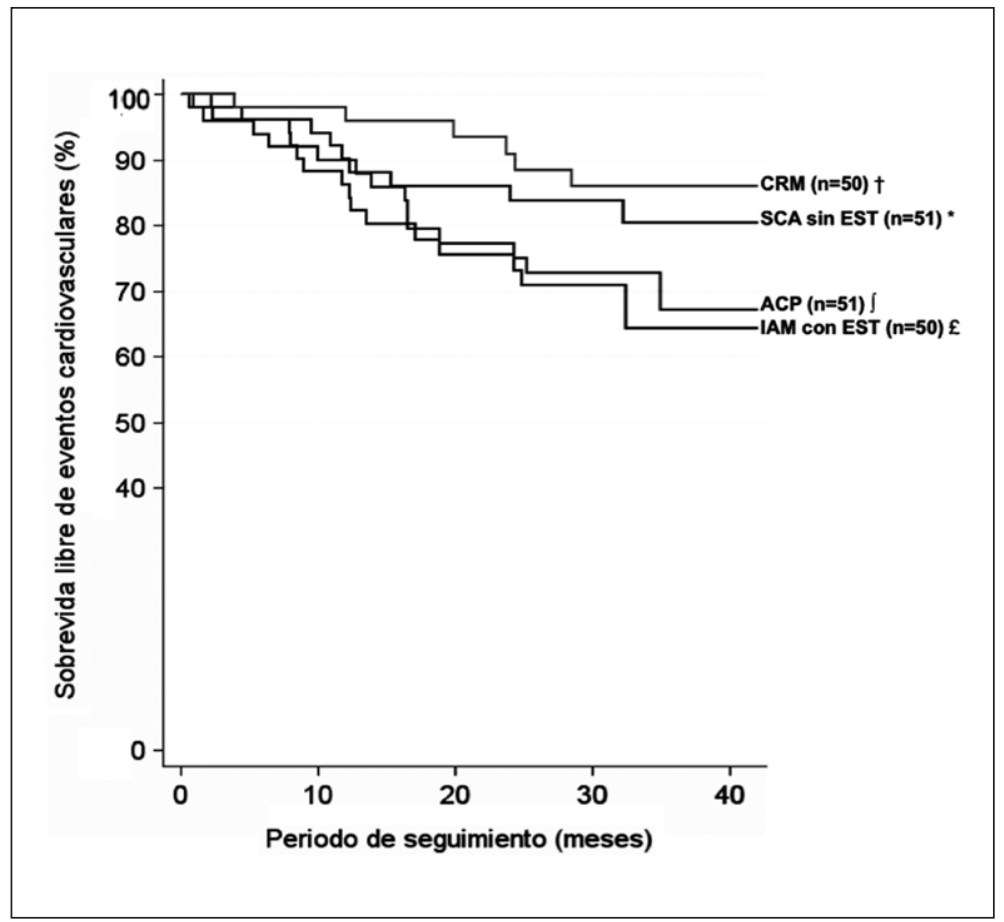

Figura 1. Sobrevida libre de eventos cardiovasculares mayores durante el período de seguimiento, según tipo de evento coronario que determino la elegibilidad. ${ }^{`} \mathrm{ACP}=$ angioplastía coronaria percutánea; ${ }^{+} \mathrm{CRM}=$ cirugía de revascularización miocárdica; ${ }^{\mathrm{f}} \mathrm{AM} \sin \mathrm{EST}$ = infarto agudo al miocardio sin elevación de segmento $\mathrm{ST}$; *SCA sin EST = síndrome coronario agudo sin elevación de segmento ST.

Tabla 5. Análisis de los factores de riesgo cardiovascular en grupo de pacientes que presentó eventos cardiovasculares mayores durante el período de seguimiento

\begin{tabular}{|c|c|c|c|}
\hline $\begin{array}{l}\text { Factores de riesgo cardiovascular } \\
\text { Promedios - frecuencia (\%) }\end{array}$ & $\begin{array}{l}\text { Grupo sin ECVM* } \\
\qquad(n=157)\end{array}$ & $\begin{array}{l}\text { Grupo con ECVM* } \\
(n=45)\end{array}$ & Valor de $p$ \\
\hline Edad (años) & $63,1 \pm 9,7$ & $66,2 \pm 8,8$ & 0,05 \\
\hline Sexo femenino & $26,7 \% \quad(42)$ & $42,2 \%(19)$ & 0,05 \\
\hline Hipertensión arterial crónica & $84,7 \%(133)$ & $88,8 \%(40)$ & 0,33 \\
\hline Antecedente de dislipidemia** & $52,2 \% \quad(82)$ & $73,3 \%(33)$ & 0,009 \\
\hline Diabetes Mellitus tipo 2 & $33,7 \% \quad(53)$ & $42,2 \%(19)$ & 0,19 \\
\hline \multicolumn{4}{|l|}{ Índice de masa corporal } \\
\hline - Normal $\left(18,5-24,9 \mathrm{~kg} / \mathrm{m}^{2}\right)$ & $15,2 \% \quad(24)$ & $24,4 \%(11)$ & 0,43 \\
\hline - Sobrepeso $\left(25,0-29,9 \mathrm{~kg} / \mathrm{m}^{2}\right)$ & $24,4 \% \quad(11)$ & $26,6 \%(12)$ & 0,39 \\
\hline - Obesidad $\quad\left(\geq 30 \mathrm{~kg} / \mathrm{m}^{2}\right)$ & $77,3 \% \quad(35)$ & $48,8 \%(22)$ & 0,39 \\
\hline Tabaquismo activo & $8,9 \% \quad(14)$ & $4,4 \% \quad(2)$ & 0,26 \\
\hline Clearence creatininemia $<60 \mathrm{ml} / \mathrm{min} / 1,73 \mathrm{~m}^{2 * * *}$ & $9,55 \%(15)$ & $15,5 \% \quad(7)$ & 0,19 \\
\hline Antecedentes familiares de enfermedad coronaria precoz ${ }^{\dagger}$ & $11,4 \% \quad(18)$ & $15,5 \% \quad(7)$ & 0,30 \\
\hline
\end{tabular}

*Eventos cardiovasculares mayores. **Antecedente de dislipidemia obtenido de registro en ficha clínica, no se registraron niveles basales de colesterol o sus fracciones. ${ }^{* * *}$ Clearence de creatininemia estimado según formula de Crockcroft y Gault. †Familiar de primer grado, menor de 55 años si es hombre o menor de 65 años si es mujer. 
Tabla 6. Eventos cardiovasculares mayores durante el período de seguimiento, posterior al evento coronario índice, que determinó la elegibilidad

\begin{tabular}{|c|c|c|}
\hline Eventos cardiovasculares mayores & Frecuencia (\%) & Tiempo presentación (meses) \\
\hline ACP electiva* & $11,3 \%(23)$ & $16,9 \pm 9,5$ \\
\hline ACP urgencia & $4,4 \% \quad(9)$ & $9,7 \pm 8,4$ \\
\hline CRM electiva ${ }^{f}$ & $3,4 \% \quad(7)$ & $13,4 \pm 10,2$ \\
\hline Nuevo IAM no fatal ${ }^{+}$ & $1,9 \% \quad(4)$ & $9,2 \pm 6,2$ \\
\hline CRM urgencia ${ }^{f}$ & $1,4 \% \quad(3)$ & $17,6 \pm 6,9$ \\
\hline ACV no fatalf & $1,4 \% \quad(3)$ & $16,3 \pm 12,2$ \\
\hline
\end{tabular}

${ }^{*} \mathrm{ACP}$ electiva = angioplastía coronaria percutánea electiva, en pacientes que durante el seguimiento presentaron angor de esfuerzo posterior al evento coronario índice; ' $\mathrm{ACP}$ urgencia = angioplastía coronaria percutánea de urgencia; ${ }^{\mathrm{f} C R M}=$ cirugía de revascularización miocárdica electiva o de urgencia; ${ }^{\dagger} \mathrm{I} M \mathrm{M}=$ infarto agudo al miocardio no fatal; ${ }^{f} \mathrm{ACV}=$ accidente cerebro vascular no fatal.

El abandono del tabaquismo en pacientes con EC está relacionado con disminución de la mortalidad e incidencia de eventos $\mathrm{CV}^{18,19}$. En nuestra serie, menos de $8 \%$ de los pacientes reporta uso de tabaco al momento del control y la tasa de abandono posterior al primer evento $\mathrm{CV}$ es de $84,4 \%$, sin embargo, no hubo una disminución significativa de la incidencia de eventos $\mathrm{CV}$, debido probablemente al número de pacientes evaluados. Los resultados son alentadores, en estudios europeos el consumo de tabaco posterior a un evento coronario es cercano a $18 \% \%^{5}$, cabe destacar que la implementación de un programa de seguimiento efectivo puede lograr reducir el tabaquismo a $<3 \%{ }^{16}$.

El manejo óptimo de la HTA reduce la incidencia de re-infarto, muerte por EC y ACV ${ }^{20-21}$. Treinta por ciento de los pacientes alcanzaron metas de PA $<130 / 85 \mathrm{mmHg}$ y sólo $23,6 \%$ de aquellos con 3 o más FR. Esto puede deberse a subutilización de los fármacos antihipertensivos, realidad observada en otras partes del mundo ${ }^{3}$, además del abandono de controles médicos y la falta de adherencia al tratamiento farmacológico.

A pesar que el pronóstico de los pacientes diabéticos post angioplastía, IAM y angina inestable es menos auspicioso ${ }^{22,23}$, en nuestro estudio no hubo diferencias en la incidencia de eventos $\mathrm{CV}$ mayores al compararlos con pacientes no diabéticos. Alrededor de $60 \%$ de los diabéticos tiene 3 o más FRCV y logran promedios de hemoglobina glicosilada $<7 \%$ en $50 \%$ de los casos, hallazgo relevante si se compara con datos de otros estudios en los cuales el promedio de hemoglobina glicosilada varía entre $7-9 \%{ }^{24-26}$.

Los fármacos de acción CV como antiagregantes plaquetarios, estatinas, beta bloqueadores y los bloqueadores del eje renina-angiotensina-aldosterona, han demostrado reducir la mortalidad en pacientes con EC, por lo tanto, la gran mayoría debería recibir estos fármacos ${ }^{27-30}$.

Encontramos una adecuada prescripción de estos fármacos. Aspirina tiene un uso cercano al 90\%, IECA/ARAII sobre 70\%, similar a lo encontrado en estudios internacionales ${ }^{5,11}$ y superior a lo observado en Latinoamérica ${ }^{3}$. Los beta bloqueadores adrenérgicos están indicados en $2 / 3$ de los pacientes, proporción menor comparado al $80 \%$ reportado por series europeas, pero mayor a lo observado en Latinoamérica que varía entre $11-45 \%{ }^{8}$.

Con respecto al uso de estatinas está indicada en 3 de 4 pacientes, comparable a países de altos ingresos ${ }^{3,5,11}$. Pese a esto sólo $2 / 3$ alcanzan CT $<200$ $\mathrm{mg} / \mathrm{dL}$, la mitad alcanza niveles de colesterol LDL $<100 \mathrm{mg} / \mathrm{dL}$, que puede deberse a tipo y dosis de estatinas disponibles. Destaca que alrededor de 90\% de los pacientes tiene niveles de colesterol HDL sobre $40 \mathrm{mg} / \mathrm{dL}$, que puede deberse al bajo punto de corte establecido en las recomendaciones nacionales ${ }^{14}$.

Al igual que en los estudios EUROASPIRE I, II y III, se aprecia que lograr un control metabólico con cambios de estilo de vida no es fácil y que el mayor impacto se logra con el uso de fármacos, situación que no deja de ser preocu- 
pante por la probabilidad de adherencia en los años venideros.

En conclusión, los datos obtenidos de forma global, no se alejan de la realidad mundial. Sin embargo, se requieren mayores esfuerzos para fortalecer las normas de manejo en pacientes coronarios e incorporar a la práctica estrategias de prevención secundaria tales como educación por enfermera previa al alta hospitalaria, reforzar el abandono del tabaquismo, monitorización de la terapia farmacológica y fomentar un estilo de vida saludable, incorporando la actividad física y una alimentación equilibrada ${ }^{21,31,32}$.

\section{Referencias}

1. Allender S, Scharbotough P, Peto V, Rayner M, Leal J, Luengo-Fernández R, et al. European Cardiovascular Disease Statistics: 2008 edition. London: British Heart Foundation; 2008.

2. Leal J, Luengo-Fernández R, Gray A, Petersen S, Rayner M. Economic burden of cardiovascular diseases in the enlarged European Union. Eur Heart J 2006; 27 (13): 1610-9.

3. Yusuf S, Islam S, Chow CK, Rangarajan S, Dagenais G, Díaz R, et al. Use of secondary prevention drugs for cardiovascular disease in the community in high-income, middle-income, and low-income countries (the PURE Study): a prospective epidemiological survey. Lancet 2011; 378 (9798): 1231-43.

4. Evolución de la mortalidad en Chile según causas de muerte y edad, 1990-2007. Instituto Nacional de Estadística (INE) - Chile. [Internet] 2009. Disponible en: http:// www.ine.cl/canales/chile_estadistico/demografia_y_vitales/estadisticas_vitales/estadisticas_vitales.php [Consultado el 1 de mayo de 2012].

5. Kotseva K, Wood D, De Backer G, De Bacquer D, Pyorala $\mathrm{K}$, Keil U, et al. Cardiovascular prevention guidelines in daily practice: a comparison of EUROASPIRE I, II, and III surveys in eight European countries. Lancet 2009; 373 (9667): 929-40.

6. Smith SC Jr, Benjamin EJ, Bonow RO, Braun LT, Creager MA, Franklin BA, et al. AHA/ACCF Secondary Prevention and Risk Reduction Therapy for Patients with Coronary and other Atherosclerotic Vascular Disease: 2011 update: a guideline from the American Heart Association and American College of Cardiology Foundation. Circulation 2011; 124 (22): 2458-73.

7. Force M, Perk J, De Backer G, Gohlke H, Graham I, Reiner Z, et al. European Guidelines on cardiovascular disease prevention in clinical practice (version 2012):
The Fifth Joint Task Force of the European Society of Cardiology and Other Societies on Cardiovascular Disease Prevention in Clinical Practice (constituted by representatives of nine societies and by invited experts) * Developed with the special contribution of the European Association for Cardiovascular Prevention \& Rehabilitation (EACPR). Eur Heart J. 2012 May 3.

8. Schargrodsky H, Hernández-Hernández R, Champagne BM, Silva H, Vinueza R, Silva Aycaguer LC, et al. CARMELA: assessment of cardiovascular risk in seven Latin American cities. Am J Med 2008; 121 (1): 58-65.

9. EUROASPIRE. A European Society of Cardiology survey of secondary prevention of coronary heart disease: principal results. EUROASPIRE Study Group. European Action on Secondary Prevention through Intervention to Reduce Events. Eur Heart J 1997; 18 (10): 1569-82.

10. Group EIS. Lifestyle and risk factor management and use of drug therapies in coronary patients from 15 countries; principal results from EUROASPIRE II Euro Heart Survey Programme. Eur Heart J. 2001 Apr; 22 (7): 554-72.

11. Kotseva K, Wood D, De Backer G, De Bacquer D, Pyorala $\mathrm{K}$, Keil U, et al. EUROASPIRE III: a survey on the lifestyle, risk factors and use of cardioprotective drug therapies in coronary patients from 22 European countries. Eur J Cardiovasc Prev Rehabil. 2009 Apr; 16 (2): 121-37.

12. Gaede P, Vedel P, Larsen N, Jensen GV, Parving HH, Pedersen O. Multifactorial intervention and cardiovascular disease in patients with type 2 diabetes. $\mathrm{N}$ Engl J Med 2003; 348 (5): 383-93.

13. Euroaspire I, Group II, European Action on Secondary Prevention by Intervention to Reduce E. Clinical reality of coronary prevention guidelines: a comparison of EUROASPIRE I and II in nine countries. EUROASPIRE I and II Group. European Action on Secondary Prevention by Intervention to Reduce Events. Lancet 2001; 357 (9261): 995-1001.

14. Programa de Salud Cardiovascular. Ministerio de SaludChile. [Internet] 2002. Disponible en: http://www.minsal.gob.cl/portal/url/page/minsalcl/g_proteccion/g_cardiovascular/presentacion_programacardiovascular.html [Consultado el 1 de mayo de 2012].

15. Lavie CJ, Milani RV, Ventura HO. Obesity and cardiovascular disease: risk factor, paradox, and impact of weight loss. J Am Coll Cardiol. 2009 May 26; 53 (21): 1925-32.

16. Guarda E, Acevedo M, Lira MT, Chamorro G, Corbalán R. [Prevalence of cardiovascular risk factors among patients suffering vascular events on admission and one year later]. Rev Med Chile 2005 Oct; 133 (10): 1147-52.

17. Lanas F, Avezum A, Bautista LE, Díaz R, Luna M, Islam 
$S$, et al. Risk factors for acute myocardial infarction in Latin America: the INTERHEART Latin American study. Circulation 2007; 115 (9): 1067-74.

18. Critchley JA, Capewell S. Smoking cessation for the secondary prevention of coronary heart disease. Cochrane Database of Systematic Reviews 2003, Issue 4.

19. Álvarez L, Balibrea J, Suriñacha M, Coll R, Pascual M, Toril J, et al. Smoking cessation and outcome in stable outpatients with coronary, cerebrovascular, or peripheral artery disease. European Journal of Cardiovascular Prevention \& Rehabilitation 0 (00) 1-10.

20. Flack JM, Neaton J, Grimm R Jr, Shih J, Cutler J, Ensrud $\mathrm{K}$, et al. Blood pressure and mortality among men with prior myocardial infarction. Multiple Risk Factor Intervention Trial Research Group. Circulation 1995; 92 (9): 2437-45.

21. Wood DA, Kotseva K, Connolly S, Jennings C, Mead A, Jones J, et al. Nurse-coordinated multidisciplinary, family-based cardiovascular disease prevention programme (EUROACTION) for patients with coronary heart disease and asymptomatic individuals at high risk of cardiovascular disease: a paired, cluster-randomised controlled trial. Lancet 2008; 371 (9629): 1999-2012.

22. Lowel H, Koenig W, Engel S, Hormann A, Keil U. The impact of diabetes mellitus on survival after myocardial infarction: can it be modified by drug treatment? Results of a population-based myocardial infarction register follow-up study. Diabetologia 2000; 43 (2): 218-26.

23. Gustafsson I, Hildebrandt P, Seibaek M, Melchior T, Torp-Pedersen C, Kober L, et al. Long-term prognosis of diabetic patients with myocardial infarction: relation to antidiabetic treatment regimen. The TRACE Study Group. Eur Heart J 2000; 21 (23): 1937-43.

24. Lowel H, Koenig W, Engel S, Hormann A, Keil U. The impact of diabetes mellitus on survival after myocardial infarction: can it be modified by drug treatment? Results of a population-based myocardial infarction register follow-up study. Diabetologia 2000; 43 (2): 218-26.
25. Gustafsson I, Hildebrandt P, Seibaek M, Melchior T, Torp-Pedersen C, Kober L, et al. Long-term prognosis of diabetic patients with myocardial infarction: relation to antidiabetic treatment regimen. The TRACE Study Group. Eur Heart J 2000; 21 (23): 1937-43.

26. Ismail-Beigi F, Craven T, Banerji MA, Basile J, Calles J, Cohen RM, et al. Effect of intensive treatment of hyperglycaemia on microvascular outcomes in type 2 diabetes: an analysis of the ACCORD randomised trial. Lancet 2010; 376 (9739): 419-30.

27. Yusuf S, Sleight P, Pogue J, Bosch J, Davies R, Dagenais G. Effects of an angiotensin-converting-enzyme inhibitor, ramipril, on cardiovascular events in high-risk patients. The Heart Outcomes Prevention Evaluation Study Investigators. N Engl J Med 2000; 342 (3): 145-53.

28. Antithrombotic Trialists C. Collaborative meta-analysis of randomised trials of antiplatelet therapy for prevention of death, myocardial infarction, and stroke in high risk patients. BMJ 2002; 324 (7329): 71-86.

29. Cannon CP, Steinberg BA, Murphy SA, Mega JL, Braunwald E. Meta-analysis of cardiovascular outcomes trials comparing intensive versus moderate statin therapy. J Am Coll Cardiol 2006; 48 (3): 438-45.

30. de Peuter OR, Lussana F, Peters RJ, Buller HR, Kamphuisen PW. A systematic review of selective and nonselective beta blockers for prevention of vascular events in patients with acute coronary syndrome or heart failure. Neth J Med 2009; 67 (9): 284-94.

31. Fonarow GC, Gawlinski A, Moughrabi S, Tillisch JH. Improved treatment of coronary heart disease by implementation of a Cardiac Hospitalization Atherosclerosis Management Program (CHAMP). Am J Cardiol 2001; 87 (7): 819-22.

32. Birtcher KK, Greisinger AJ, Brehm BJ, Wehmanen OA, Furman LM, Salinas CC, et al. A secondary prevention lipid clinic reaches low-density lipoprotein cholesterol goals more often than usual cardiology care with coronary heart disease. J Clin Lipidol 2010; 4 (1): 46-52. 